DEBATE

\title{
Paradojas, controversias, discurso y realidad del tabaquismo en Cuba
}

\author{
Paradoxes, controversies, speeches and realities of smoking in \\ Cuba
}

\section{Nery Suárez Lugo}

Licenciada en Psicología. Doctor en Ciencias de la Salud. Investigador Titular. Escuela Nacional de Salud Pública. La Habana, Cuba.

\begin{abstract}
RESUMEN
El conocimiento de la epidemia del tabaquismo y su evolución, tienen que ser el punto de partida de los programas de prevención y control. En Cuba existe un total conocimiento de su magnitud, se dispone de un Programa de Prevención y Control del Tabaquismo concebido con carácter nacional, sistemático, integral, interdisciplinario e intersectorial, pero la ejecución no marcha acorde con lo que se quiere alcanzar en la salud de la población. Las causas son conocidas, pero para su análisis hay que centrarse en las paradojas y controversias en relación con el consumo de tabaco, que tienen como resultado la brecha existente en el discurso y la realidad. La situación de país productor, el arraigo del hábito, su vinculación a la vida cotidiana, cultural y política, hacen que el cubano se considere un pueblo de fumadores, de ahí que la aceptación social del tabaquismo, conspire con la aplicación de medidas de control aunque estas se encuentren definidas en las políticas públicas. La salud pública tiene un importante reto, pues la situación que se muestra hoy día en relación con otros países impone que se asuma con total responsabilidad la política y estrategia documentada, para hacerla coherente con el accionar de cada día en todas las instancias del Sistema Nacional de Salud, para lograr la integración e integralidad que se plantea.
\end{abstract}

Palabras clave: Tabaquismo, epidemia, prevalencia, consumo.

\section{ABSTRACT}


Knowing the smoking epidemics and its progression must be the starting point of the prevention and control programs. In Cuba, there is wide knowledge of the dimension of the problem and also a National Smoking Prevention and Control Program that is comprehensive, systematic, interdisciplinary and intersectoral; however, the implementation of this program does not agree with what must be achieved in terms of the population's health. The causes are known, but one must focus on the paradoxes and controversies around smoking in Cuba for their analysis, which results in the existing gap between the speech and the reality. The fact that Cuba is a tobacco producer, the deep-rooted smoking habit and linking to the daily, cultural and political life make the Cubans think that the Cuban people is a population of smokers; hence, the social acceptance of smoking goes against the implementation of control measures, even though they are well defined in the public policies. The Cuban public health faces a significant challenge because the present situation compared to that of other countries demands that we must be full responsible for the documented policy and strategy, to make them agree with the everyday work at all levels of the national healthcare system and attain the intended integration and integrality.

Key words: Smoking, epidemics, prevalence, consumption.

\section{LA EPIDEMIA TABÁQUICA}

Aunque no siempre resulta posible establecer de manera exacta la fase en que se encuentra un determinado país en relación con la evolución de la epidemia tabáquica según la propuesta de Allan López, ${ }^{1}$ lo cierto es que el modelo resulta de utilidad para enmarcar el momento de acuerdo al patrón de consumo de tabaco en la población y en función de ello establecer la estrategia adecuada para poder avanzar en función de lograr su erradicación.

La prevalencia del hábito de fumar en Cuba ha sido considerada alta desde que se dispone de información al respecto. Algunos estudios indicativos realizados antes de 1970 informan cifras superiores al 60 \% en la población total.

Los primeros datos referentes a la prevalencia nacional del tabaquismo en Cuba datan del año 1978, de una encuesta realizada por el Ministerio de Comercio Interior, en la cual 68,9\% de la población de 17 años y más fumaba.

Posteriormente el Instituto Cubano de Investigaciones y Orientación de la Demanda Interna realiza en el año 1980 una encuesta nacional, y obtiene una prevalencia de $52,9 \%$. Lo anterior pone de manifiesto que hasta el inicio de la década del 80 el país se encontraba en la primera fase de la epidemia.

La elevada prevalencia y consumo per cápita anual de cigarrillos que ubica a Cuba en los primeros lugares a nivel regional y mundial, hacen que se inicien en la segunda mitad de la década del 80 del pasado siglo acciones nacionales con carácter sistemático y enfoque intersectorial. ${ }^{2}$

En 1984 se encuentra una prevalencia de $42,2 \%$ y el consumo per cápita anual de cigarrillos era de 2 470; en 1988 la prevalencia desciende a 40,1\% y el consumo per cápita anual de cigarrillos a 2 106. Son fumadores $47,3 \%$ de los hombres y 
$25,5 \%$ de las mujeres, por lo que es posible considerar que el inicio del programa antitabaco se enmarca en la segunda fase de la epidemia. ${ }^{3}$

En el 1995 fumaba $36,8 \%$ de la población cubana, 48,1\% de los hombres y 26,3 $\%$ de las mujeres, por lo que a pesar de las acciones que se realizaban, la epidemia continuaba en la segunda fase y no se obtenían los resultados requeridos. ${ }^{4}$

A escala nacional se estimó que en el 2001 existían 2047714 fumadores de 17 años y más, lo que representó una prevalencia de $31,9 \%$, con un decrecimiento en la prevalencia general tanto en hombres, $(41,4 \%)$ como mujeres $(23,0 \%)$.

La iniciación global en el grupo de edad 20 a 24 años fue $22,2 \%$ y la prevalencia en adolescentes que han consumido cigarrillos alguna vez de $25 \%{ }^{5}$ Sin embargo, se obtuvieron cifras superiores en estudiantes de 13 a 15 años de edad $(29,9 \%){ }^{6}$

Tomando como base esas cifras, entre 78 países miembros de la Organización Mundial de la Salud, Cuba ocupa el lugar $28 .^{7}$

También en el año 2001, el consumo per cápita anual fue estimado en 1314 cigarrillos, representando también disminución en relación con años anteriores. ${ }^{8}$ Tal como describe la literatura, aproximadamente a los 25 años transcurridos desde el inicio de la epidemia, se transita hacia la tercera fase. ${ }^{9}$

Hasta el año 2001, desde el inicio del Programa de Prevención y Control del Tabaquismo, se logró una tendencia a la disminución de prevalencia y consumo, discreta, pero sostenida. ${ }^{10}$

De las defunciones ocurridas en 1995 y 2007, se atribuyeron al tabaquismo 15 y 18 $\%$ de muertes prevenibles, respectivamente. En Cuba, en el año 2007, el tabaquismo causó $86 \%$ de las defunciones por cáncer de pulmón, 78 \% de las muertes por enfermedad pulmonar obstructiva crónica, $28 \%$ de la mortalidad por cardiopatía isquémica y el $26 \%$ de las defunciones por enfermedad cerebrovascular. ${ }^{11}$

El consumo per cápita anual de cigarrillos estimado en el año 2009 fue $1548 .^{12}$ En lo que respecta a la prevalencia no se disponen cifras actualizadas de encuestas nacionales, pero la información disponible permite hacer algunas estimaciones.

En los seis años transcurridos de 1995 al 2001, la prevalencia ha descendido 4,9\% (36,8 \% vs. 31,9\%) y el consumo per cápita anual de cigarrillos 5,81\% (1 395 vs. $1314)$. Situación similar debe presentarse en el momento actual, por lo que si el consumo se ha incrementado en este período de ocho años en una cifra de 17,80 $\%$, es de esperar que la prevalencia también lo hiciera.

Un estudio realizado con una muestra representativa del municipio Playa de La Habana en el año 2007, registra una prevalencia de $38,2 \%$ muy similar a la encontrada en el año $2001 .^{13}$

Igual tendencia que siguen las fases de la epidemia, presenta la situación de la mortalidad, en un período de 20 a 30 años más tarde, por lo que el análisis anterior hace pensar que no resultará posible reducirla en aquellas enfermedades que hoy constituyen las primeras causas de muertes y que se encuentran asociadas al consumo de tabaco. 
El conocimiento de la epidemia y su evolución tienen que ser el punto de partida de los programas de prevención y control, pero lamentablemente, aunque a nivel mundial, regional y por supuesto local, existe un total conocimiento de su magnitud, la ejecución de estos no marcha acorde con lo que se quiere alcanzar en la salud de la población.

Esto hace, que como en el caso de Cuba, la epidemia se detenga en la fase tercera y que resulte difícil avanzar hacia la reducción del tabaquismo, no solo lograr que los fumadores cesen, sino que la incidencia sea cada vez menor para que no se produzca un reemplazo de los fumadores y con ello dar continuidad, de generación en generación a este comportamiento tan nocivo a la salud.

Las causas pueden también ser conocidas, pero no por ello hay que dejar de insistir en su análisis y difusión, de ahí que este artículo se centre en las paradojas y controversias en relación con el consumo de tabaco en Cuba que tienen como resultado la brecha existente entre el discurso y la realidad.

\section{LAS PARADOJAS}

En los países productores de tabaco, la situación del tabaquismo se torna más compleja, pues la aceptación social es mayor y se une a ello el hecho de que el tabaco es una importante fuente de empleo y sustento económico. Por su papel como producto de exportación, constituye también fuente de ingreso en moneda convertible, lo que hace que se presente la situación paradójica de resultar importante cultivar tabaco y a la vez promover salud, en países como Cuba, donde ambos empeños los asume el Estado.

Esta paradoja se refleja en el medio social, donde la cultura y el folklore hacen presente el cigarro en la música, bailes y donde particularmente el tabaco forma parte de los rituales religiosos, como ofrenda a los dioses o simplemente para acompañar la danza y otros espectáculos. ${ }^{14}$

A la vez, los lugares de recreación y muy especialmente los centros nocturnos, además de expender cigarrillos, se asocian a su consumo, pues una nube de humo rodea las pistas de baile, las mesas de los asistentes y al propio escenario y sus artistas. Fumar ha sido relacionado de manera directa a la diversión, la recreación y el esparcimiento. Una noche de cabaret, de discoteca o salón de fiestas, representa una importante cantidad de humo de segunda mano para los que no fuman y muchas colillas en el cenicero de aquellos que si lo hacen.

Cuba se identifica como la tierra del ron, el tabaco y la música, derivado de una época en que la caña era el principal cultivo y las hojas del tabaco estaban catalogadas como de las mejores del mundo, y ante poco que exhibir, la música cubana se incluía en la imagen de este país empobrecido y explotado como otros muchos.

La realidad cambió y hoy existen otras muchas cosas que mostrar, pero sigue siendo esa la imagen que se promueve al turismo y que por consiguiente se trasmite a los propios cubanos, que a la vez se convierten en emisores de esa información. 
Sin embargo, el conocimiento de la nocividad del tabaco a la salud y la toma de conciencia a nivel social, han llevado a que en ese mismo medio social se promueva un estilo de vida sano.

Esto hace que el cubano, tanto niño como adulto, se mueva en un medio social donde de manera clara se manifiesta la paradójica situación de enaltecer y reprobar el acto de fumar, donde los medios de comunicación resaltan la importancia del cultivo de la planta para la economía, mientras que antes, de manera casi simultánea o poco después, se presentan programas dedicados a promover la salud, o se proyectan mensajes donde se destaca la nocividad del tabaquismo. Algo similar ocurre en la radio.

En la prensa plana, no ha dejado de suceder que en una página se señale la relación de fumar con la mortalidad por cáncer o infarto, mientras que la siguiente presenta a un anciano, próximo a cumplir el siglo de vida, aspirando el humo de su tabaco.

Un ejemplo final puede ilustrar de manera muy clara la situación. El día 29 de mayo se celebra en Cuba el "Día del Trabajador Tabacalero". Por la importancia de la actividad y por el hecho de haber sido seleccionado ese día en particular por ser el aniversario del nacimiento de Lázaro Peña, uno de los líderes más prestigiosos de la clase obrera cubana, todos los medios de difusión se encargan de destacar la fecha, se hacen múltiples reportajes a los trabajadores del sector tabacalero en todo el proceso productivo y de comercialización. El día 31 de mayo es el "Día mundial sin fumar" y entonces comienzan los mensajes antitabaco, que aunque generalmente con menor amplitud e intensidad, ocupan los espacios en que antes se hablara de las bondades del tabaco.

En la Encuesta Mundial de jóvenes se encontraron resultados que demuestran lo anterior, 92,4\% de los estudiantes de secundaria básica encuestados vio mensajes contra el cigarrillo en los medios de comunicación, lo cual es más acentuado entre varones que en las hembras. (94,5 vs. 90,2 \%).

También se muestra que más del $60 \%$ de los estudiantes refieren haber visto a su vez mensajes a favor del tabaco, $62,9 \%$ de los no fumadores y $67,9 \%$ del los fumadores. Predomina significativamente en los jóvenes fumadores activos, poseer algún objeto alegórico al cigarrillo que en aquellos que nunca han fumado. Esta característica aumenta en los varones de octavo grado. Más estudiantes fumadores activos $(13,7 \%)$, manifestaron que algún representante de entidades comercializadoras del producto, le han ofrecido un cigarrillo gratis en relación con los no fumadores y fundamentalmente los de octavo grado $(20,3 \%)$ sin diferencias significativas de sexo. ${ }^{15}$

No distante de todo lo anterior se encuentra la presencia de líderes de opinión fumadores, algunos de los cuales a la vez educan en la importancia de no fumar, situación que se encuentra en los maestros, los profesionales sanitarios y en los padres de familia.

Encuestas nacionales realizadas han obtenido cifras de prevalencia de fumadores de $25 \%$ para los médico/as y $29 \%$ para las enfermero/as. En los maestros la cifra se aproxima al $30 \%$ En el año 1990 el maestro fue referido como patrón imitativo para fumar por $83,2 \%$ de los encuestados, los padres por $56,9 \%$, las madres por $34,7 \%$, y los amigos solo por $31,3 \% .^{5}$

Estudios realizados en hospitales han obtenido cifras superiores, pues se detectó entre médico(as) y enfermero(as) una prevalencia de $33 \%$ y $47 \%$

http://scielo.sld.cu 
respectivamente. Un estudio reciente realizado en la atención primaria encuentra prevalencia de $38,4 \%$ enfermero(as), 35,6 \% médicos(as) y 33,3\% estomatólogos(as). ${ }^{16}$

Aunque en encuestas nacionales los médicos(as), enfermeros(as) y maestros(as) fuman con frecuencias inferiores a las de la población general, estas son consideradas altas si se tiene en cuenta su papel de educadores y modelos sociales.

La exposición al humo ambiental del tabaco en Cuba es alta: más de la mitad de las familias cubanas, $55 \%$ de los niños, $51 \%$ de las embarazadas y $60 \%$ de los adolescentes están expuestos a este agente contaminante, lo que nos ubica en el lugar 22 de los países de mayor prevalencia de tabaquismo pasivo en el hogar, de los 98 que enviaron datos a la Organización Mundial de la Salud y el tercer lugar de los 26 países de América que informaron este dato, superado por Argentina (70\%) y Uruguay $(63 \%) .{ }^{17}$ Sin embargo, este último país se declaró en el año 2006 primer país de Las Américas totalmente libre de humo de tabaco (ambientes sanitarios y educativos y oficinas públicas $100 \%$ libres de humo de tabaco en todo el país), por lo que la medidas legales implementadas permitieron dar una respuesta para solucionar lo encontrado. ${ }^{18}$

\section{LAS CONTROVERSIAS}

Todos los años el "Festival del Habano" centra la atención de los medios de comunicación, tan lucida actividad se desarrolla con la presencia de figuras muy reconocidas de ese mundo que se reúnen para festejar con todos los recursos que tan lucrativa empresa permite.

Las subastas que se realizan han permitido obtener recursos para emplear en la salud, lo que no ha resultado bien visto por los que se encargan de la salud pública, pues existen ideas contrapuestas al respecto. Si bien los recursos se emplean en una causa de bien público, no es menos cierto que van a reparar los propios daños que causan, pero no comparables con los beneficios que obtienen.

El laureado Ballet Nacional de Cuba, en una ocasión apoyó este Festival, con la puesta en escena de la obra "Romeo y Julieta" para servir en ese momento de promoción al lanzamiento de la marca de habanos de ese nombre, haciendo el juego a la tan discutida participación de los elementos de la cultura en la promoción del tabaquismo, que por demás resulta tan distante de lo que es el estilo de vida de los bailarines.

Es este el debate de un problema no resuelto y para el que los salubristas no hemos sido capaces de aportar la información requerida que pueda sustentar, desde el punto de vista económico, que en un país como Cuba, donde el Estado asume los gastos en que incurren los fumadores para atender su salud, la relación costo-beneficio de tener en el tabaco un importante rubro de exportación, no es tan beneficiosa como parece al analizar solo las cifras de lo que aporta, sin reducir los gastos que genera a esa propia economía.

Otro punto de controversia está centrado en la legislación, cuya efectividad demostrada la convierte en elemento central que pudiera unirse a la educación y sinergizar el deseado efecto de reducir el tabaquismo. 
Las regulaciones y prohibiciones de fumar en Cuba han tenido un lento movimiento, no solo porque lograr aprobaciones de estos rangos requiere su tiempo, sino porque no ha existido el apoyo requerido para que se logre.

Aunque algunos sugieren la aplicación de medidas no tan drásticas a través de restricciones parciales, alegando que de esta manera se da tiempo a la preparación de los fumadores para el cambio, sin embargo, es reconocido que el tabaquismo pone en peligro la salud de toda la comunidad $y$, sobre todo, la salud de los que no fuman y comparten el mismo ambiente cerrado con los fumadores.

Para informar y educar se obtiene fácil el consenso, pero al hablar de prohibir siempre surgen los defensores de la libertad individual y en esa controversia de proteger al fumador pasivo y agredir al fumador, hace lento el proceso de aprobación de todo documento legal.

Cuba no ha sido de los países más aventajados en la presencia de figuras legales que regulen y prohíban fumar en lugares públicos y otras medidas de carácter económico cuyo sustento sea también jurídico, a pesar que el Programa de Prevención y Control del Tabaquismo en su fundamento y plan lo establece a través de regulaciones y medidas, no solo por el Ministerio de Salud Pública, sino también por otros ministerios implicados en este problema.

En el 1988 se inició un proceso para conformar y elevar una propuesta de documento legal, con rango de Decreto Ley, que aunara todo lo ya establecido en relación con regulaciones y prohibiciones de fumar, a la vez que se propusiera incrementar el control mediante la imposición de sanciones. Un estudio realizado en ese año muestra que $78 \%$ de la población considera insuficientes las prohibiciones y regulaciones establecidas en lugares públicos y $76 \%$, que no se cumplen las implantadas. Estos argumentos permitieron fundamentar la primera propuesta, que comenzó a promoverse y conciliarse ese año, fue entregada a las instancias correspondientes del gobierno, pero no logró su cometido. ${ }^{19}$

Han transcurrido más de 20 años y no solo no ha sido alcanzada esa meta, sino que el cumplimiento de las regulaciones existentes tiene aún un tímido grado de aplicación y discreto cumplimiento.

En la actualidad, el Acuerdo 5570 del 2005, del Comité Ejecutivo del Consejo de Ministros (CECM), es el documento legal de mayor fuerza y alcance, que plantea la prohibición de la venta de cigarrillos y tabacos a menores de 18 años de edad, establece la prohibición de fumar en locales públicos cerrados que no tengan áreas creadas a tales efectos, así como en los medios masivos de transportación; establece también la prohibición de fumar en los centros e instituciones educacionales, de la salud e instalaciones deportivas, en los que, de considerarse por los organismos correspondientes, se habilitarán áreas específicas para fumar. En los demás centros e instalaciones pertenecientes a otros organismos o instituciones se crearán áreas para fumar. En cualquier caso, las áreas de fumadores y de no fumadores deberán estar debidamente señalizadas. ${ }^{20}$

Un estudio realizado en el municipio Habana del Este de Ciudad de la Habana, en el año 2007, puso en evidencia que la aplicación y cumplimiento de las regulaciones de venta y prohibiciones de fumar en lugares públicos, como está establecido en el Acuerdo, es insuficiente, siendo los principales factores asociados a la no aplicación y cumplimiento, la falta de exigencia, control y la poca importancia que le confieren los directivos y autoridades de salud de las localidades correspondientes. 
El insuficiente cumplimiento del Acuerdo 5570 de 2005 del CECM, derivado de la falta de exigencia y control de las autoridades competentes en lo referente a la comercialización de los cigarrillos, constituye un elemento a favor de la accesibilidad del producto. ${ }^{21}$

La presencia de los cigarrillos entre los productos normados de la canasta familiar que se ofertan a la población a precios diferenciados, es también el reflejo de las controversias en lo que se refiere a ser considerada como un consumo dañino y perjudicial a la salud que sin embargo, se incluye dentro de aquellos que son priorizados para satisfacer necesidades básicas de la población.

En el 2009, de cada 100 cigarrillos que se vendieron, 24 corresponden al mercado de precios bajos y 76 al de precios altos, de ellos, 65 se compran en moneda nacional y 11 en libremente convertible. Esta información, no difiere de la que se ha ofrecido de manera reiterada como resultado de esos estudios, siendo de gran importancia a los efectos de poder definir una política de precios coherente con la intención de reducir el consumo de este nocivo producto.

Sin embargo, se mantiene la oferta en moneda nacional de cigarrillos sueltos en la red de comercio minorista, con su correspondiente efecto de estimular el consumo en los jóvenes al abaratar su precio. Continúa la oferta de cajetillas con 10 cigarrillos para la venta en pesos convertibles, que como estrategia de mercadeo, cumple el mismo propósito.

Las ventas de cigarrillos se desplazan de una forma de comercialización a otra, por lo que puede disponerse de un pronóstico de reducción a partir del análisis de la proporción de cada forma de comercialización.

El precio promedio de la cajetilla de cigarrillos en el año 2009 fue de 7,15 pesos cubanos. Se redujo 0,15 pesos cubanos por cajetilla en relación con el año anterior. La equivalencia del precio promedio es de 0,30 pesos cubanos convertibles (CUC) y 0,36 dólares estadounidenses (USD). El porcentaje de cambio en el precio promedio del cigarrillo, al comparar el año 2009 con el año 2008, decreció 2,73 $\% .^{22}$

La política actual de precios y comercialización de los cigarrillos, no constituye un mecanismo desestimulador del consumo, la distribución gratuita que realizan algunas entidades explica en parte la situación contradictoria dada en el 2009 de que un fumador de una cajetilla diaria invierte al mes 215 pesos cubanos, la mitad del salario medio del país (427 pesos cubanos). ${ }^{23}$

\section{DISCURSO Y REALIDAD}

Mientras no se resuelvan las paradojas y controversias anteriormente señaladas, el discurso y la realidad no van a marchar de la mano y por consiguiente se mantiene la brecha entre lo que está previsto hacer y lo que realmente se hace, teniendo como resultado que no se va a lograr que el tabaquismo deje de ser esa epidemia silenciosa que disminuye los logros en salud que hoy tenemos, afecte la calidad de vida de los cubanos y se siga trasmitiendo de generación en generación como parte de nuestras costumbres y tradiciones que han hecho que el cubano se identifique a si mismo como "un pueblo de fumadores." ${ }^{24}$ 
En las proyecciones de la salud pública para el 2015, en el acápite Factores Relacionados con el Comportamiento, en tabaquismo, se plantean 5 propósitos con sus metas a alcanzar, tanto intermedia en este año 2010 como para el 2015, las cuáles han tomado para la línea de base los diferentes documentos que han sido analizados con anterioridad:

Como primer propósito, se encuentra reducir en $40 \%$ la prevalencia de fumadores en la población cubana. La meta prevista para el año 2010 es que la prevalencia nacional sea 25,6 \% y que para el año 2015 se reduzca a 19,2 \%, tomando como línea de base $32 \%$ del 2001.

Como segundo propósito se plantea reducir la prevalencia en los adolescentes que han consumido cigarrillos alguna vez, planteando como meta que esta sea 20 $\%$ en el 2010 y $15 \%$ en el 2015, tomando como línea de base $25 \%$.

El propósito 3 se refiere a los profesionales de la salud y lograr reducir en $60 \%$ la prevalencia de fumadores. Como meta intermedia se plantea llegar a $20 \%$ en el 2010, tomando $31 \%$ como línea de base.

El propósito 4 está dirigido a reducir en $12 \%$ el tabaquismo pasivo teniendo como meta lograr $7 \%$ de reducción en el 2010, tomando como línea de base $88 \%$.

El propósito 5 es reducir hasta $20 \%$ en el año 2015, la iniciación global en el grupo de 20 a 24 años teniendo como meta intermedia para el 2010 una reducción a $21 \% .^{25}$

El Programa Nacional de Prevención y Control del Tabaquismo y su plan de acción integral, contempla los lineamientos para igual período, reflejando iguales propósitos, metas, estrategias y acciones que permitirán obtener tales resultados. ${ }^{26}$

Como se puede observar, los documentos rectores están en total correspondencia con la experiencia nacional e internacional en lo relacionado a cuánto y cómo reducir el tabaquismo, sin embargo, la brecha entre lo que se describe en el discurso y la realidad aún es suficientemente amplia.

En el 2007 en un municipio de la capital del país, se realizó una investigación para determinar los conocimientos, actitudes y prácticas de los médicos de los consultorios tipo I y enfermeros de los consultorios tipo II sobre las actividades de promoción de salud y prevención de enfermedades relacionadas con el tabaquismo, presentes en los programas nacionales vigentes en el Ministerio de Salud Pública.

Se localizaron los programas nacionales disponibles; se seleccionaron aquellos que debían contener actividades de promoción de salud y prevención de enfermedades relacionadas con el tabaquismo. Se realizó la revisión del Programa Nacional de Prevención y Control del tabaquismo vigente en esos momentos y se determinaron las acciones referidas anteriormente que debían ser efectuadas en la atención primaria de salud.

Con los conocimientos aportados por el análisis de este programa, se revisaron los programas que debían contener actividades relacionadas con la promoción y prevención del tabaquismo, se seleccionaron aquellos donde estaban presentes estas acciones y se identificaron las acciones que debían ser ejecutadas en ese nivel de atención. 
Algunos programas de control de enfermedades relacionadas con el tabaquismo no incluyen acciones de promoción y prevención contra esta adicción, a pesar que se ha considerado como un factor de riesgo muy importante para diferentes enfermedades. La mayoría de los programas donde están presentes estas acciones resultan generales, insuficientes, inespecíficas y no uniformes.

El Programa Nacional de Prevención y Control del Tabaquismo, en esencia presenta un enfoque de promoción de salud, ofrece las condiciones necesarias para que reciban tratamiento y rehabilitación las personas que lo soliciten, indica un soporte legal para proteger la salud del fumador y del no fumador, principalmente los niños, niñas, adolescentes y jóvenes, con base en la investigación científica que conlleva a decisiones acertadas en la política y estrategia trazada.

Se obtuvieron resultados que mostraron, como en otros estudios, la elevada prevalencia de fumadores entre los directivos, médicos(as) y enfermeros(as), pero lo fundamental, que una cifra importante considera no efectivas las acciones que se desarrollaban en el área de salud para la erradicación del tabaquismo. ${ }^{27}$

La propuesta de Decreto Ley sobre la Prevención y el Control del Tabaquismo transita de nuevo por los canales administrativos establecidos para poder ser analizada y aprobada. Queda menos tiempo para actuar si realmente se quiere reducir la morbilidad y la mortalidad en aquellas enfermedades que hoy se reflejan en el cuadro de salud. ${ }^{28}$

La realidad es evidente para cualquier ciudadano que transite por nuestros escenarios comunes, en los centros de estudio, trabajo y salud, por citar los más importantes, se sigue fumando, los adolescentes y jóvenes comienzan, el tabaquismo no ha perdido la aceptación social a pesar de tantos años sin publicidad directa y tanto empeño en lograr estilos de vida que tributen a la salud.

La salud pública tiene un importante reto, pues la situación que se muestra hoy día en relación con otros países, ${ }^{29}$ impone que se asuma con total responsabilidad la política y estrategia documentada para hacerla coherente con el accionar de cada día en todas las instancias del Sistema Nacional de Salud, para lograr la integración e integralidad que se plantea y su enfoque intersectorial.

Cerrar la brecha en Cuba, donde el tabaco es parte de la propia vida, es bien difícil pero no imposible, solo se trata de lograr que el equilibrio en esa paradoja haga que el enfoque de salud predomine, que seamos capaces de fundamentar de manera científica, la relación costo beneficio para que pueda prevalecer la salud pública en esa perenne controversia, entonces y solo de esa forma, la realidad de acercará al discurso y fumar no formará parte del estilo de vida del cubano de estos tiempos, sin dejar de considerar al tabaco, como parte de nuestra historia.

\section{REFERENCIAS BIBLIOGRÁFICAS}

1. López AD, Collishaw NE, Piha T. A descriptive model of the cigarette epidemic in developed countries. Tobacco Control. 1994;3:242-7.

2. Instituto Cubano de Investigaciones y Orientación de la Demanda Interna. Programa para la prevención y control del tabaquismo. La Habana: ICIODI; 1988. 
3. International Organization of Consumer Union. Tobacco Control in the Third World Atlas 1990. Penang (Mal): IOCU;1990.

4. Instituto Nacional de Higiene y Epidemiología. Encuesta nacional de factores de riesgo. Cuba 1995. La Habana: INHEM, MINSAP; 1995.

5. Instituto Nacional de Higiene, Epidemiología y Microbiología. MINSAP. Encuesta nacional de factores de riesgo. Cuba 2000- 2001. La Habana: INHEM; 2002.

6. Lancés CL. Primera encuesta mundial sobre tabaquismo en jóvenes. Cuba 2001. Informe investigación. La Habana: INHEM, ENSAP, MINSAP; 2001.

7. Mackay J, Eriksen M, Shafey O. The Tobacco Atlas. 2da ed. American Cancer Society. World Lung Foundation. London (UK): Myraid; 2006.

8. Suárez LN. Consumo, precio y comercialización de los productos manufacturados del tabaco en Cuba. Rev Cubana Salud Pública 2006;32(2) Disponible en: http://bvs.sld.cu/revistas/spu/vol32 2 06/spu02206.htm

9. Fernández E, Villalbí JR, Córdoba R. Lecciones aprendidas en el control del tabaquismo en España. Salud Pública Mex. 2006;48(1).

10. Suárez Lugo N. Tabaco o salud: una decisión social. Horizontes Sanitarios. México. UJAT. 2004;3(2):12-21.

11. Varona PP, Herrera TD, García RGR, Bonet GM, Romero PT, Venero FST Mortalidad atribuible al tabaquismo en Cuba. Rev Cubana Salud Pública. $2009 ; 35(2)$.

12. Suárez Lugo N. Estudio sobre consumo de productos manufacturados del tabaco. Cuba. 2010. Informe de investigación. La Habana: Escuela Nacional de Salud Pública. MINSAP; 2010.

13. Suárez Lugo N, Campos Pupo E. Prevalencia y percepción del riesgo del tabaquismo. Área de salud del Policlínico Dr. Jorge Ruiz Ramírez, Ciudad de La Habana, 2007. Rev Cubana Salud Pública. 2010;36(2).

14. Suárez Lugo N. Enfoque Social de la Mercadotecnia Sanitaria. La Habana: Editorial Ciencias Médicas; 2007.

15. Lancés CL. Segunda encuesta mundial sobre tabaquismo en jóvenes. Cuba 2004. La Habana: INHEM, ENSAP, MINSAP; 2005.

16. Suárez LN. Negrín ES, Caraballoso HM. El tabaquismo en los profesionales de la salud. Habana Vieja, 2007. Rev Cubana Salud Pública. 2008;34(4):1-11.

17. Varona PP. Informe técnico sobre tabaquismo. Año 2006. La Habana: Instituto de Higiene, Epidemiología y Microbiología, MINSAP;2006.

18. Blanco MA. Seis años que cambiaron el control del tabaco en Uruguay: lecciones aprendidas. Washington, D.C.: OPS; 2007.

19. Pinillos AL, Suárez Lugo N. Programa para la prevención y control del tabaquismo en Cuba. En: Mujer y tabaquismo. Lima: Ed. Art Director, E.I.R.L; 1995.

http://scielo.sld.cu 
20. Comité Ejecutivo del Consejo de Ministros República de Cuba. Acuerdo 5570. Ciudad de La Habana: CECM; 2 de diciembre de 2005.

21. Suárez Lugo N, Rodríguez CDM, Caraballoso HM. Legislación para el control del tabaquismo en Cuba. Rev Cubana Salud Pública. 2008;34(3).

22. Suárez Lugo N. El consumo de productos manufacturados del tabaco en Cuba. Rev Cubana Salud Pública. 2006;32(2):1-17.

23. Oficina Nacional de Estadísticas. Anuario Estadística 2008. Edición 2009 [sitio en Internet]. 2009 [citado 12 Feb 2010]. Disponible en: http://www.one.cu/aec2008.htm

24. Suárez Lugo N. América latina: los jóvenes y el tabaquismo. Revista Promotion Edu J Health. 1995;2(1):52-4.

25. Ministerio de Salud Pública. Proyecciones de la salud pública en Cuba para el año 2015. La Habana: Editorial Ciencias Médicas; 2006.

26. Ministerio de Salud Pública de Cuba. Programa para la prevención y control del tabaquismo. Lineamientos 2008-2015. La Habana: MINSAP; 2009.

27. Acosta COS. Conocimiento, actitudes y prácticas sobre prevención del tabaquismo en la atención primaria de salud. Municipio Diez de Octubre. Ciudad de la Habana. 2007 [tesis]. La Habana: Escuela Nacional de Salud Pública; 2009.

28. Ministerio de Salud Pública. Situación de salud en Cuba. 2008. Indicadores Básicos. Dirección Nacional de Registros Médicos y Estadística de Salud. La Habana: MINSAP; 2009.

29. Mackay J, Eriksen M, Shafey O, Ross H. The Tobacco Atlas. 3th ed. Atlanta, Georgia (USA): American Cancer Society, World Lung Foundation; 2009.

Recibido: 16 de febrero de 2010.

Aprobado: 30 de julio de 2010.

Nery Suárez Lugo. Escuela Nacional de Salud Pública. Calle Línea esq. I. El Vedado 10400. La Habana, Cuba. Correo electrónico: nerysl@infomed.sld.cu 